\title{
Microarray analysis in acute and chronic hepatitis B virus infection Yongwei $\mathrm{Li}^{* 1,2}$, Mingfen $\mathrm{Zhu}^{3}$ and Gang $\mathrm{Li}^{1}$
}

Address: ${ }^{1}$ Department of Infectious Diseases, the Third Affiliated Hospital of Sun Yat-sen University, Guangzhou, PR China, ${ }^{2}$ Department of Traditional Chinese Medicine, the Third Affiliated Hospital of Sun Yat-sen University, Guangzhou, PR China and ${ }^{3}$ Department of Infectious Diseases, the Sixth Hospital of Shanghai Jiao Tong University, Shanghai, PR China

* Corresponding author

from Frontiers of Retrovirology: Complex retroviruses, retroelements and their hosts Montpellier, France. 21-23 September 2009

Published: 24 September 2009

Retrovirology 2009, 6(Suppl 2):P50 doi:10.1 186/1742-4690-6-S2-P50

This abstract is available from: http://www.retrovirology.com/content/6/S2/P50

(c) 2009 Li et al; licensee BioMed Central Ltd.

\section{Background}

Different genes are involved in the pathogenesis of acute and chronic hepatitis B virus (HBV) infection, although little is known about these genes.

\section{Materials and methods}

A full length HBV genome was cloned and sequenced from serum of a chronic hepatitis $\mathrm{B}(\mathrm{CHB})$ patient. The genome and sterilized Milli-Q water were transfected to HepG2 and HepG2.2.15 cells, respectively, by lipofectamine 2000. The prior cell line was named HepG29BL. The two cell lines were collected post transfection 48 hours. Differential genes were examined using Affy Human U133 2.0A gene chip; Real time polymerase chain (RT-PCR) was performed to confirm the expression of lumican in six human hepatoma carcinoma cell lines.

\section{Results}

Of 50,000 transcripts and variants on the gene chip, 4860 genes were significantly altered between the two cell lines. Pro-inflammatory and interferon associated molecules (e.g, IL-6, IFNAR2, IFNA17, etc.) were up regulated in HepG29BL, while down regulated in HepG2.2.15. The ratio of lumican was significantly high between the two cell lines. The levels of lumican were showed in six cell lines as follows: HepG29BL> HepG2>7402> Alexander>MHCC-97 > HepG2.2.15 $(\mathrm{P}<0.05$, but the comparsion between MHCC-97 and HepG2.2.15 was not statistically significant).

\section{Conclusion}

The result of this study provides a foundation for future investigations of genes involved in the pathogenesis of HBV infection. Lumican, as reported in CHB [1] and in other organs cancer pathogenesis study [2], may play an role in liver fibrosis and carcinogenesis.

\section{Acknowledgements}

We would like to thank Advanced Throughput (Shanghai), Inc for technical support.

\section{References}

I. Keiko FuruTai, Sayaka Sato, Taeko Yamauchi, et al: Intrahepatic gene expression profiles in chronic hepatitis B and autoimmune liver disease. J Gastroenterol 2008, 43:866-874.

2. Li Y, Aoki T, Mori Y, Ahmad M, Takino T, Sato H, et al.: Cleavage of lumican by membrane-type matrix metalloproteinase-I abrogates this proteoglycma-mediated suppression of tumor cell colony formation soft agar. Cancer Res 2004, 64:7058-7064. 